\title{
Anaesthesia and Anaesthetics
}

\section{Case Report}

\section{Transversus abdominis plane catheter for nephrectomy in a patient with lupus: a case report}

\author{
Brian C Martin MD* \\ Baystate Medical Center, Department of Anesthesiology, UMASS Medical School 759 Chestnut St. Springfield, MA 01199, USA
}

\begin{abstract}
A patient with systemic lupus erythematosus and thrombocytopenia, presented for an open right nephrectomy for a recurrent right renal hemorrhagic cyst. The patient was deemed unsuitable to receive a thoracic epidural catheter due to low platelets. We decided to perform a single shot right-sided transversus abdominis plane block prior to incision. We then asked the surgeon to place a right transversus abdominis plane catheter under direct visualization. The patient experienced effective pain control during her hospital stay and consumed a relatively low dose of opioids.
\end{abstract}

\section{Introduction}

Transversus abdominis plane (TAP) blockade may provide effective analgesia in patients undergoing abdominal surgery $[1,2]$. Thoracolumbar fibers ranging from T7-L1, which innervate the abdominal wall, can be anesthetized. ${ }^{3}$ TAP catheters can be inserted, providing some analgesia when compared to thoracic epidural analgesia (TEA) and can be a viable option when contraindications to TEA exist $[3,4]$.

A patient with systemiclupus erythematosus and thrombocytopenia, presented for an open right nephrectomy due to a hemorrhagic renal cyst. A single shot right-sided lateral to medial TAP block was performed and then a TAP catheter was inserted.

Written consent was obtained from the patient for publication of this case report.

\section{Case description}

A 42 year-old-female, weighing $54 \mathrm{~kg}$, with a history or systemic lupus erythematous (SLE), resulting thrombocytopenia and end stage renal disease on peritoneal dialysis, presented for a right nephrectomy for recurrent hemorrhagic cyst. During the preoperative assessment, it was noted the patient's platelet count was $79 \times 10^{3} /$ microliter. After a discussion with the surgical team, we consented the patient for a rightsided transversus abdominis plane block and catheter. After induction of general anesthesia with an endotracheal tube, we performed a single shot right TAP block, with the use of a B Braun 22 gauge, 2 inch needle under ultrasound guidance, prior to incision. The injectate included $30 \mathrm{ml}$ of $0.25 \%$ bupivacaine with epinephrine 1:200,000, $50 \mathrm{mcg}$ of dexmedetomidine and dexamethasone $4 \mathrm{mg}$. The TAP block was performed at the level of the umbilicus, in the right mid-axillary line between the iliac crest and the subcostal margin. During the operation, the patient received fentanyl $250 \mathrm{mcg}$ as the only opioid. Towards the end of the surgical procedure, the surgeon placed a B. Braun, 20 gauge, 40 -inch polyamide catheter between the internal oblique and transversus abdominis muscles. The catheter was placed at the T10-11 dermatomes. The catheter was sutured to the skin after being tunneled via the abdominal wall, laterally.
The patient was extubated and taken to the post anesthesia care unit after the procedure. The patient reported a pain score of $5 / 10$ and received a $10 \mathrm{ml}$ bolus of $1 \%$ lidocaine, via the TAP catheter. The patient's pain improved and appeared to have decreased sensation to touch in the anterior T10-12 dermatomes, bilaterally. The catheter was connected to an infusion pump containing $0.1 \%$ bupivacaine at $10 \mathrm{ml}$ an hour, which infused for approximately 68 hours and was discontinued on post op day 3 . She also received nurse administered boluses of intravenous hydromorphone in the post anesthesia care unit followed by intravenous patient controlled hydromorphone. She received a total of $1.8 \mathrm{mg}$ of intravenous hydromorphone. Only 0.4 $\mathrm{mg}$ of hydromorphone was self-administered. Once the TAP catheter infusion was discontinued, she was given Tylenol (acetaminophen) with codeine pills $(300 \mathrm{mg} / 30 \mathrm{mg})$. She received a total of 4 pills containing acetaminophen and codeine. The patient was hospitalized for 5 days. During this period, the average pain score was $3 / 10$, ranging from $0-6 / 10$. The most commonly reported pain score was $0 / 10$. The patient began to ambulate on post-operative day 2. Ambulation was delayed due to nausea and pain at the left peritoneal dialysis catheter site. She was discharged on post-operative day 4 with good pain control and no obvious complications from the TAP catheter were noted as the catheter site appeared clean dry and intact, without signs of bleeding or infection.

\section{Discussion}

This is the first known report of a TAP catheter, placed in lieu of an epidural catheter, due to thrombocytopenia, in a patient with SLE. The transversus abdominis plane is a muscular anatomical plane between the internal oblique and the transversus abdominis muscles $[1,3]$. Thoracolumbar nerve fibers from T7-L1 may be present in this plane, as demonstrated in cadaver and imaging studies [1,3]. Introducing

Correspondence to: Brian C Martin MD, Baystate Medical Center, Department of Anesthesiology, UMASS Medical School 759 Chestnut St. Springfield, MA 01199, USA, E-mail: Brian.Martin@baystatehealth.org

Received: September 26, 2017; Accepted: October 18, 2017; Published: October 20,2017 
local anesthetic into this plane, depending on the anatomical site between the iliac crest and the subcostal margin, will anesthetize the overlying skin, muscle and parietal peritoneum and potentially reduce opioid consumption $[1,5]$.

TAP blocks have been utilized in other surgeries, to include hysterectomy, hernia repair, peritoneal dialysis catheter placement and renal transplant $[1,6]$. Johns et al, in a systematic review, concluded that a TAP block does significantly reduce opioid consumption, although there was no difference in pain scores when compared to placebo [3]. There is variability with regard to the analgesic effect of a TAP block based on the anatomic spread of the injectate and the location of the block. Injection cephalad to iliac crest, in the mid-axillary line, as performed in this case, may be ideal for an incision in the T10-L1 dermatomes [1]. In renal surgery, avoidance of intraperitoneal structures eliminates the visceral pain component [1]. This may suggest that the TAP block may eliminate a majority of the pain and serve as the primary anesthetic, as discussed by Henshaw et al. [6]

Although thoracic epidural anesthesia is the mainstay of analgesia for abdominal surgery [3], the TAP block can serve as a viable alternative, especially when a neuraxial technique is not feasible $[1,4]$. Some authors have reported a thoracic epidural failure rate as high as $27 \%$ [3]. As with many pain procedures, inadequate analgesia is a complication, and combined with the risk of neuraxial bleeding, the risk/benefit discussion may change, regarding thoracic epidural analgesia. Alcock et al reported the successful and safe use of TAP catheters in 2 coagulopathic patients [4]. Some studies have also demonstrated that epidural analgesia may lead to higher failure rates than TAP blocks or equal analgesia [3].

Patients with systemic lupus erythematosus frequently have hematologic abnormalities. These include anemia, leukopenia and thrombocytopenia [7]. A review of the patient's medical record revealed that she did have chronic thrombocytopenia. She was also on dialysis, another risk factor for platelet dysfunction and possibly neuraxial complications [1]. If there is no visceral pain component, a TAP catheter, as part of a multimodal analgesic plan may suffice. Performing a TAP block and catheters may avoid some of the side effects and complications seen with epidural anesthesia such as hypotension and neuraxial bleeding [1].

The additives for the TAP block included dexmedetomidine and dexamethasone, as well as epinephrine. In a recent review article by Kirksey et al., these additives were examined. Dexmedetomidine was shown to consistently prolong the sensory blockade for peripheral nerve blocks. The evidence for dexamethasone and epinephrine were inconsistent but their use was supported by some studies [8]. It is possible that with a catheter placed in a linear incision, targeting 1-2 dermatomes, that the pain relief would have been effective without the additives. However, given the variability of pain relief with TAP blocks, and the fact that the patient did have pain, highlight an important aspect of the use of the TAP catheter: to reduce opioid consumption. This patient appeared to have effective analgesia based on her pain scores and opioid consumption.

Her ambulation on post-operative day 1 was delayed due to pain at her dialysis catheter site and nausea during peritoneal dialysis. However, on post-operative day 2-4 she ambulated many times, tolerated a regular diet and had normal bowel function.

In conclusion, a TAP catheter can be an invaluable analgesic modality, as it may reduce opioid consumption, serving as an alternative to neuraxial techniques in patients at risk for bleeding complications.

\section{Financial disclosures}

\section{None}

\section{Conflicts of interest}

\section{None}

\section{References}

1. Farag E, Guirgis MN, Helou M, Dalton JE, Ngo F, et al. (2015) Continuous transversus abdominis plane block catheter analgesia for postoperative pain control in renal transplant. J Anesth 29: 4-8. [Crossref]

2. Johns N, O'Neill S, Ventham NT, Barron F, Brady RR, et al. (2012) Clinical effectiveness of transversus abdominis plane (TAP) block in abdominal surgery: a systematic review and meta-analysis. Colorectal Dis 14: e635-642. [Crossref]

3. Ganapathy S, Sondekoppam RV, Terlecki M, Brookes J, Adhikary SD, et al. (2015) Comparison of efficacy and safety of lateral-to-medial continuous transversus abdominis plane block with thoracic epidural analgesia in patients undergoing abdominal surgery: A randomized, open-label feasibility study. Eur J Anaesthesiol 32: 797-804. [Crossref]

4. Allcock E, Spencer E, Frazer, R, Applegate G, Buckenmaier C (2010) Continuous Transversus Abdominis Plane (TAP) Block Catheters in a Combat. Pain Med 11: 14261429. [Crossref]

5. Klasen F, Bourgoin A, Antonini F, Dazeas E2, Bretelle F, et al. (2016) Postoperative analgesia after caesarean section with transversus abdominis plane block or continuous infiltration wound catheter: A randomised clinical trial. TAP vs. infiltration after caesarean section. Anaesth Crit Care Pain Med 35: 401-406. [Crossref]

6. Henshaw D, Baker ML, Weller RS, Reynolds JW, Jaffe JD (2016) Transversus abdominis plane block as the primary anesthetic for peritoneal dialysis catheter surgery. J Clin Anesth 31: 182-188. [Crossref]

7. Aleem A, Al Arfaj AS, khalil N, Alarfaj H (2014) Haematological abnormalities in systemic lupus erythematosus. Acta Reumatol Port 39: 236-341. [Crossref]

8. Kirksey MA, Haskins SC, Cheng J, Liu SS (2015) Local Anesthetic Peripheral Nerve Block Adjuvants for Prolongation of Analgesia: A Systematic Qualitative Review. PLoS One 10: e0137312. [Crossref]

Copyright: (C2017 Martin BC. This is an open-access article distributed under the terms of the Creative Commons Attribution License, which permits unrestricted use, distribution, and reproduction in any medium, provided the original author and source are credited. 